Pacific Journal of Mathematics

CENTERS OF REGULAR SELF-INJECTIVE RINGS 


\title{
CENTERS OF REGULAR SELF-INJECTIVE RINGS
}

\author{
K. R. GOODEARL
}

This paper is concerned with calculating centers of regular self-injective rings, particularly those obtained by completions with respect to rank functions, and those obtained as factor rings of other regular self-injective rings. Sufficient conditions are developed under which the completion of a regular ring $R$ has the same center as $R$. For any regular self-injective ring $R$ of Type $I_{f}$, it is shown that the center of any factor ring of $R$ is a factor ring of the center of $R$. These results are used to distinguish among the simple regular self-injective rings of Type $\mathrm{II}_{f}$ by their possible centers.

All rings in this paper are associative with unit, and all ring maps are assumed to preserve the unit.

1. Introduction. The class of regular, right self-injective rings may be divided into subclasses using the theory of types as in [6, Chapters 5-7]. In particular, any indecomposable, regular, right self-injective ring must be one of Types $\mathrm{I}_{f}, \mathrm{I}_{\infty}, \mathrm{II}_{f}, \mathrm{II}_{\infty}$, or III [6, Corollary 7.6]. The indecomposable, regular, right selfinjective rings of Types $I_{f}$ and $I_{\infty}$ are easy enough to describe, since those of Type $I_{f}$ are the simple artinian rings, while those of Type $I_{\infty}$ are the endomorphism rings of infinite-dimensional right vector spaces [6, Theorem 5.4].

In the remaining cases, however, very little is known. The center suggests itself as a reasonable invariant with which to distinguish among different rings of the same type, particularly in the indecomposable case, where the center is a field. In this paper, we develop some techniques for calculating centers, and we apply these techniques to the standard Type II examples (described below). In particular, we show that any field can be the center of a simple, regular, right and left self-injective ring of Type II, and that the standard Type II examples can be distinguished by means of their centers.

Both of the standard Type II examples are built up from certain sequences of simple artinian rings, one by the completion of a direct limit, the other by a factor ring of the direct product. The second of these is the easiest to describe, as follows. Let $R_{1}, R_{2}, \cdots$ be simple artinian rings whose composition series lengths are unbounded, and set $R=\Pi R_{n}$. If $M$ is any maximal two-sided ideal of $R$ which contains $\oplus R_{n}$, then it follows from [6, Corollary 
11.10] that $R / M$ is a simple, regular, right and left self-injective ring of Type II.

To describe the other example, we outline the completion process as developed in $[8,4,5]$. A pseudo-rank function on a regular ring $R[4$, p. 269] is a map $N: R \rightarrow[0,1]$ such that

(a) $N(1)=1$.

(b) $N(x y) \leqq N(x), N(y)$ for all $x, y \in R$.

(c) $N(e+f)=N(e)+N(f)$ for all orthogonal idempotents $e$, $f \in R$.

A rank function on $R[13, \mathrm{p} .231]$ is a pseudo-rank function $N$ such that

(d) $N(x)>0$ for all nonzero $x \in R$.

It follows from (b) and (c) that $N(x+y) \leqq N(x)+N(y)$ for all $x, y \in R$ [13, Corollary, p. 231].

Any pseudo-rank function $N$ on $R$ induces a pseudo-metric $\delta$ on $R$ according to the rule $\delta(x, y)=N(x-y)$, and the ring operations on $R$ are uniformly continuous with respect to $\delta$ [13, pp. 231, 232]. Thus the (Hausdorff) completion of $R$ with respect to $\delta$ is a ring $\bar{R}$, which we refer to as the $N$-completion of $R$. Note that the kernel of the natural map $R \rightarrow \bar{R}$ equals the kernel of $N$. According to [8, Theorem 3.7], $\bar{R}$ is a regular ring, $N$ extends continuously to a rank function $\bar{N}$ on $\bar{R}$, and $\bar{R}$ is complete with respect to $\bar{N}$. Also, $\bar{R}$ is right and left self-injective by [4, Corollary 15].

Given a simple regular ring $R$ with a rank function $N$, the $N$-completion $\bar{R}$ of $R$ need not be a simple ring or even indecomposable, as [4, Examples B, C] show. Necessary and sufficient conditions for $\bar{R}$ to be simple are given in [4, Corollary 20]. In particular, [4, Corollary 21] says that if $N$ is unique then $\bar{R}$ is simple.

The remaining Type II example is now constructed as in the following proposition.

Proposition 1.1. Let $R_{1} \rightarrow R_{2} \rightarrow \cdots$ be a sequence of simple artinian rings and ring maps such that the composition series lengths of the $R_{n}$ are unbounded, and set $R=\lim R_{n}$. Then there exists a unique rank function $N$ on $R$, and $t \overrightarrow{h e} N$-completion of $R$ is a simple, regular, right and left self-injective ring of Type II.

Proof. According to [4, Proposition 2], there is a unique rank function on each $R_{n}$. Inasmuch as rank functions on $R$ are induced by compatible rank functions on the $R_{n}$, it follows that there is a unique rank function $N$ on $R$.

Let $\bar{R}$ denote the $N$-completion of $R$. Then $\bar{R}$ is regular by 
[8, Theorem 3.7] and [4, Corollary 15] says that $\bar{R}$ is right and left self-injective. Since $N$ is unique, [4, Corollary 21] says that $\bar{R}$ is simple.

Inasmuch as the composition series lengths of the $R_{n}$ are unbounded, we infer that there exists an infinite sequence of nonzero orthogonal idempotents in $R$. Since $R$ is simple, the natural map $R \rightarrow \bar{R}$ is injective, hence $\bar{R}$ contains an infinite sequence of nonzero orthogonal idempotents. Thus $\bar{R}$ is not artinian. Consequently, we conclude as in $[6, \mathrm{pp} .33,34]$ that $\bar{R}$ is Type II.

We conclude this section by showing that any field can be the center of a regular, right self-injective ring of Type III. This result was proved by Handelman after seeing a preliminary version of this paper.

Proposition 1.2 (Handelman). Let $F$ be any field. Then there exists a simple, regular, right self-injective ring $R$ of Type III such that $\operatorname{center}(R) \cong F$.

Proof. Set $S_{n}=M_{2^{n}}(F)$ for all $n=1,2 \cdots$ Map each $S_{n} \rightarrow S_{n+1}$ along the diagonal, i.e., map $x \mapsto\left[\begin{array}{ll}x & 0 \\ 0 & x\end{array}\right]$, and set $S=\lim _{\rightarrow} S_{n}$. Note that $S$ is a simple regular ring, and that center $(S) \cong \vec{F}$.

Now let $R$ be the maximal right quotient ring of $S$. Since $S$ is a simple regular ring, we see that $R$ is a simple, regular, right self-injective ring. Given any $x \in \operatorname{center}(R)$, we see that $J=$ $\{s \in S \mid x s \in S\}$ is a nonzero two-sided ideal of $S$, whence $J=S$ and so $x \in S$. Consequently, center $(R)=\operatorname{center}(S) \cong F$.

In [7, Example (e), pp. 831, 832], it is shown that $R$ is directly infinite. Since $R$ is simple, it follows that $R$ contains no nonzero directly finite idempotents. Therefore $R$ is Type III.

2. Completions. This section is concerned with calculating the center of the completion $\bar{R}$ of a regular ring $R$ with respect to a pseudo-rank function, and in particular with conditions which ensure that the center of $\bar{R}$ coincides with the center of $R$. As our methods deal with direct limits of semisimple (artinian) rings, we begin with the case where $R$ equals the ring $M_{n}(D)$ of all $n \times n$ matrices over a division ring $D$. Our method, which is an extension of [9, Theorem 5], involves comparing the ranks of additive commutators $x y-y x$ with the ranks of differences $x-z$, where $z \in$ center $(R)$. (By the rank of a matrix $x \in M_{n}(D)$, we mean the number of linearly independent rows (or columns) of $x$.)

In order to construct additive commutators in $M_{n}(D)$ with 
suitably large ranks, we use the rational canonical form for matrices in $M_{n}(D)$, which works as well over division rings as over fields $\left[10\right.$, p. 50]. Specifically, any matrix in $M_{n}(D)$ is similar to a block diagonal matrix where each block is a companion matrix, i.e., a matrix of the form

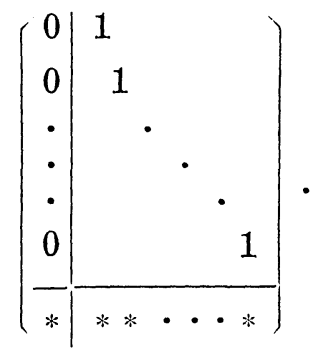

Lemma 2.1. Let $D$ be a division ring, let $n \geqq 2$ be an integer, and let $x \in M_{n}(D)$ be a companion matrix. Then there exists $y \in$ $M_{n}(D)$ such that $x y-y x$ is invertible.

Proof. If $n$ is even, set

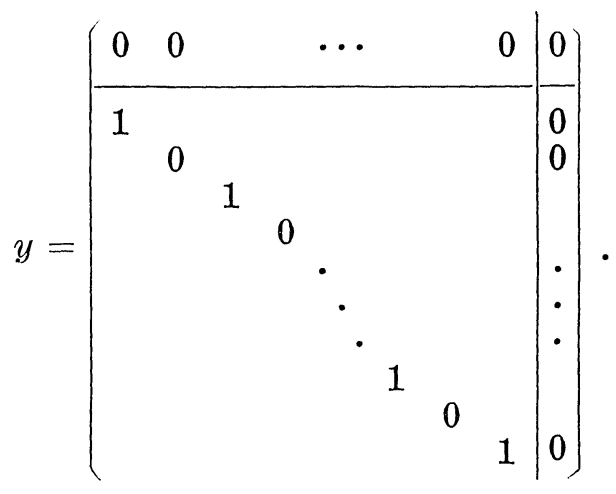

In this case, we compute that $x y-y x$ has the form

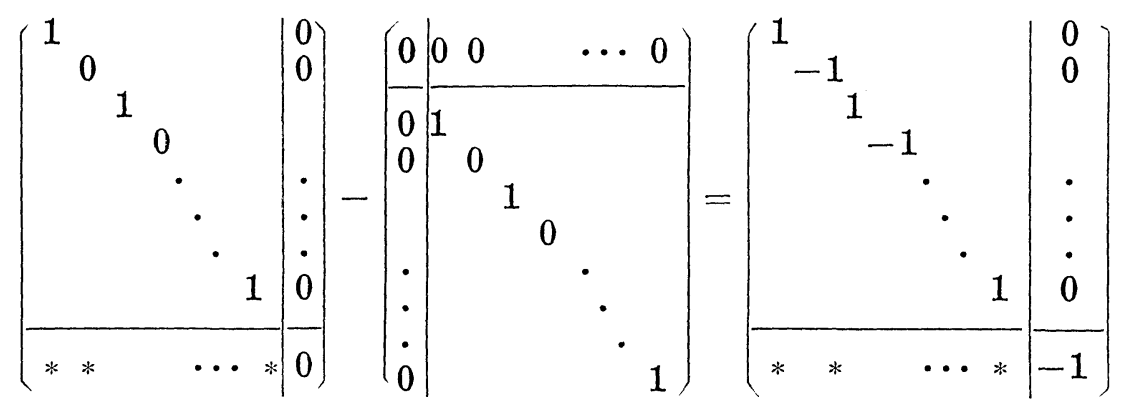


which is clearly invertible.

If $n$ is odd, set

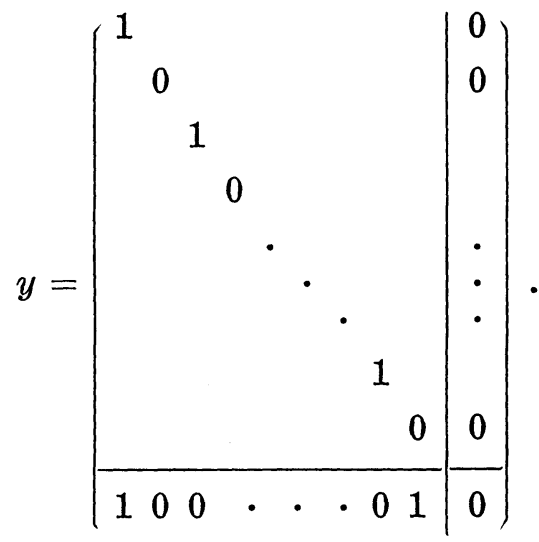

In this case, we compute that $x y-y x$ has the form

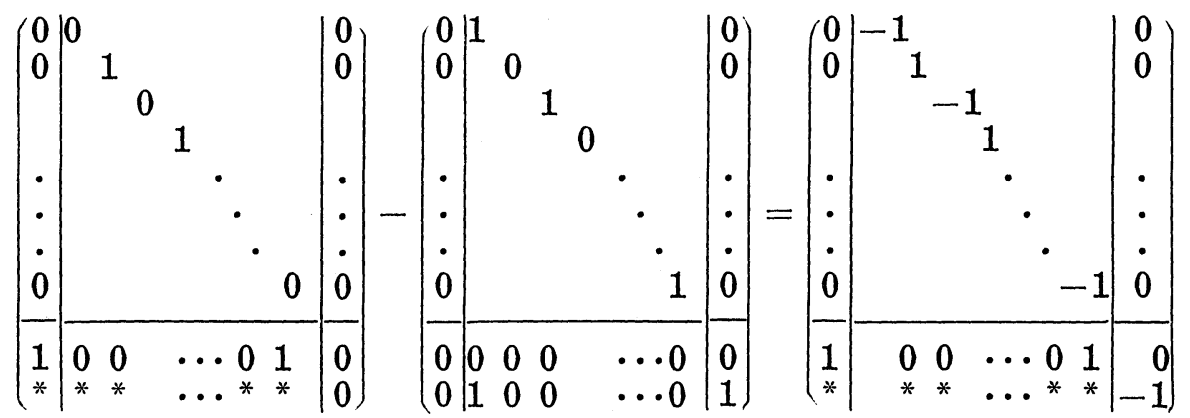

which we see is invertible (rearrange the columns in the order $2,3, \cdots, n-1,1, n$ to obtain a triangular matrix).

LEMMA 2.2. Let $D$ be a division ring, and let $n$ be a positive integer. Let $x \in M_{n}(D)$ be a diagonal matrix with diagonal entries $a_{1}, b_{1}, a_{2}, b_{2}, \cdots, a_{t}, b_{t}, a_{t+1}, a_{t+1}, \cdots, a_{t+1}$ such that $a_{i} \neq b_{i}$ for all $i=1$, $\cdots, t$.

(a) If $a_{t+1} \notin$ center $(D)$, then there exists $y \in M_{n}(D)$ such that $x y-y x$ is invertible.

(b) If $a_{t+1} \in$ center $(D)$, then there exists $y \in M_{n}(D)$ such that $\operatorname{rank}(x y-y x)=2 t$.

Proof. It clearly suffices to show that if $z=\left[\begin{array}{ll}a & 0 \\ 0 & b\end{array}\right] \in M_{2}(D)$ with $a \neq b$, then there exists $w \in M_{2}(D)$ such that $z w-w z$ is invertible. For this we need only take $w=\left[\begin{array}{ll}0 & 1 \\ 1 & 0\end{array}\right]$, since then $z w-w z=$ 
$\left[\begin{array}{ccc}0 & a-b \\ b-a & 0\end{array}\right]$

Proposition 2.3. Let $D$ be a division ring, let $n$ be a positive integer, and let $x \in M_{n}(D)$. Then there exist matrices $y \in M_{n}(D)$ and $z \in \operatorname{center}\left(M_{n}(D)\right)$ such that $\operatorname{rank}(x-z) \leqq \operatorname{rank}(x y-y x)$.

Proof. By a change of basis, we may assume that $x$ is in rational canonical form. Then, by a permutation of the basis, we may put $x$ in the form $\left[\begin{array}{cc}x^{\prime} & 0 \\ 0 & x^{\prime \prime}\end{array}\right]$, where $x^{\prime}$ is an $n^{\prime} \times n^{\prime}$ diagonal matrix and $x^{\prime \prime}$ is an $n^{\prime \prime} \times n^{\prime \prime}$ block diagonal matrix whose diagonal blocks are companion matrices of degree at least 2 . In view of Lemma 2.1, there exists an $n^{\prime \prime} \times n^{\prime \prime}$ matrix $y^{\prime \prime}$ over $D$ such that $x^{\prime \prime} y^{\prime \prime}-y^{\prime \prime} x^{\prime \prime}$ is invertible.

We may arrange the diagonal entries of $x^{\prime}$ in the order $a_{1}, b_{1}$, $a_{2}, b_{2}, \cdots, a_{t}, b_{t}, a_{t+1}, a_{t+1}, \cdots, a_{t+1}$ with $a_{i} \neq b_{i}$ for all $i=1, \cdots, t$. If $a_{t+1} \notin \operatorname{center}(D)$, then by Lemma 2.2 there exists an $n^{\prime} \times n^{\prime}$ matrix $y^{\prime}$ over $D$ such that $x^{\prime} y^{\prime}-y^{\prime} x^{\prime}$ is invertible. In this case, set $y=$ $\left[\begin{array}{rr}y^{\prime} & 0 \\ 0 & y^{\prime \prime}\end{array}\right]$ in $M_{n}(D)$, so that $x y-y x$ is invertible. Setting $z=0$, we obtain $\operatorname{rank}(x-z) \leqq n=\operatorname{rank}(x y-y x)$, as desired.

Now assume that $a_{t+1} \in \operatorname{center}(D)$, and define $z \in \operatorname{center}\left(M_{n}(D)\right)$ to be the diagonal matrix with all diagonal entries equal to $a_{t+1}$. Note that $\operatorname{rank}(x-z) \leqq 2 t+n^{\prime \prime}$. According to Lemma 2.2, there exists an $n^{\prime} \times n^{\prime}$ matrix $y^{\prime}$ over $D$ such that $\operatorname{rank}\left(x^{\prime} y^{\prime}-y^{\prime} x^{\prime}\right)=2 t$. Setting $y=\left[\begin{array}{rr}y^{\prime} & 0 \\ 0 & y^{\prime \prime}\end{array}\right]$ in $M_{n}(D)$, we conclude that $\operatorname{rank}(x y-y x)=2 t+n^{\prime \prime} \geqq$ $\operatorname{rank}(x-z)$, as desired.

Definition. Given modules $A$ and $B$, we write $A \lesssim B$ to mean that $A$ is isomorphic to a submodule of $B$. Given elements $x$ and $y$ in a regular ring $R$, we note that $x R \lesssim y R$ if and only if $x=$ $a y b$ for some $a, b \in R$.

CoROLlaRY 2.4. Let $R$ be a semisimple ring, and let $x \in R$. Then there exist elements $y \in R$ and $z \in \operatorname{center}(R)$ such that $(x-z) R \lesssim$ $(x y-y x) R$.

Proof. It suffices to consider the case when $R$ is simple artinian, hence we may assume that $R=M_{n}(D)$ for some positive integer $n$ and some division ring $D$. According to Proposition 2.3, there exist $y \in R$ and $z \in \operatorname{center}(R)$ such that $\operatorname{rank}(x-z) \leqq \operatorname{rank}(x y-y x)$. As a result, we see that the composition series length of $(x-z) R$ is less than or equal to the composition series length of $(x y-y x) R$. Consequently, we conclude that $(x-z) R \lesssim(x y-y x) R$. 
COROLLARY 2.5. Let $R$ be a directed union of semisimple subrings $R_{i}$, and assume that center $\left(R_{i}\right) \subseteq \operatorname{center}\left(R_{j}\right)$ whenever $R_{i} \subseteq R_{j}$. Given any $x \in R$, there exist elements $y \in R$ and $z \in \operatorname{center}(R)$ such that $(x-z) R \lesssim(x y-y x) R$.

Proof. We have $x \in R_{k}$ for some $k$, hence by Corollary 2.4 there exist $y \in R_{k}$ and $z \in \operatorname{center}\left(R_{k}\right)$ such that $(x-z) R_{k} \lesssim(x y-y x) R_{k}$. Since center $\left(R_{i}\right) \leqq \operatorname{center}\left(R_{j}\right)$ whenever $R_{i} \leqq R_{j}$, we see that $z \in$ center $(R)$. Inasmuch as $R$ is a flat left $R_{k}$-module, we conclude that $(x-z) R \lesssim(x y-y x) R$.

THEOREM 2.6. Let $R$ be a directed union of semisimple subrings $R_{i}$, and assume that center $\left(R_{i}\right) \subseteq \operatorname{center}\left(R_{j}\right)$ whenever $R_{i} \subseteq R_{j}$. Let $N$ be a pseudo-rank function on $R$, let $\bar{R}$ denote the $N$-completion of $R$, and let $\phi: R \rightarrow \bar{R}$ be the natural map. Then $\phi(\operatorname{center}(R))$ is dense in center $(\bar{R})$.

Proof. Let $\bar{N}$ be the natural extension of $N$ to $\bar{R}$. Let $x \in$ center $(\bar{R})$, and let $\varepsilon>0$ be a real number.

Choose $w \in R$ such that $\bar{N}(\phi(w)-x)<\varepsilon / 3$. According to Corollary 2.5, there exist $y \in R$ and $z \in \operatorname{center}(R)$ such that $(w-z) R \lesssim$ $(w y-y w) R$. Then $w-z=a(w y-y w) b$ for some $a, b \in R$, from which we see that $N(w-z) \leqq N(w y-y w)$. Since $x$ commutes with $\phi(y)$, we obtain

$$
\begin{aligned}
N(w-z) & \leqq N(w y-y w)=\bar{N}(\phi(w) \phi(y)-\phi(y) \phi(w)) \\
& =\bar{N}((\phi(w)-x) \phi(y)-\phi(y)(\phi(w)-x)) \\
& \leqq 2 \bar{N}(\phi(w)-x)<2 \varepsilon / 3
\end{aligned}
$$

and consequently

$$
\begin{aligned}
\bar{N}(\phi(z)-x) & \leqq \bar{N}(\phi(z)-\phi(w))+\bar{N}(\phi(w)-x) \\
& <N(w-z)+(\varepsilon / 3)<\varepsilon .
\end{aligned}
$$

Therefore $\phi($ center $(R))$ is dense in center $(\bar{R})$.

CoRollary 2.7. Let $R$ be a directed union of semisimple subrings $R_{i}$, and assume that center $\left(R_{i}\right) \subseteq \operatorname{center}\left(R_{j}\right)$ whenever $R_{i} \subseteq R_{j}$. Let $N$ be a rank function on $R$, let $\bar{R}$ denote the $N$-completion of $R$, and assume that $\bar{R}$ is indecomposable (as a ring). Then the natural map center $(R) \rightarrow \operatorname{center}(\bar{R})$ is an isomorphism.

Proof. Since $N$ is a rank function, we see that the natural 
map $\phi: R \rightarrow \bar{R}$ must be injective. Let $\bar{N}$ be the natural extension of $N$ to $\bar{R}$. According to Theorem 2.6, $\phi(\operatorname{center}(R))$ is dense in center $(\bar{R})$ in the $\bar{N}$-metric. Since $\bar{R}$ is an indecomposable regular ring, center $(\bar{R})$ is a field. Then for any $x \in \operatorname{center}(\bar{R})$, either $x=0$ or $x$ is invertible, hence either $\bar{N}(x)=0$ or $\bar{N}(x)=1$. Thus the $\bar{N}$ metric on center $(\bar{R})$ is discrete, hence we conclude that $\phi(\operatorname{center}(R))=$ center $(\bar{R})$. Therefore $\phi$ restricts to an isomorphism of center $(R)$ onto center $(\bar{R})$.

Corollary 2.7 does not remain valid without the compatibility condition on the centers of the $R_{i}$, as Example 2.10 shows.

The calculation of the center of the completion of a direct limit of semisimple rings with respect to a rank function was first performed by Alexander in the following special case [1, Theorem 12.8], using a fairly involved procedure. Specializing this case to the situation where $D$ is a field of characteristic zero, Handelman developed a relatively short proof in [9, Proposition 6].

THEOREM 2.8. Let $D$ be a division ring, let $n(1)<n(2)<\cdots$ be positive integers such that $n(k) \mid n(k+1)$ for all $k$, and set $R_{k}=$ $M_{n(k)}(D)$ for all $k$. Map each $R_{k} \rightarrow R_{k+1}$ by the obvious block diagonal map, and set $R=\lim R_{k}$. Then

(a) There exists a unique rank function $N$ on $R$.

(b) The $N$-completion $\vec{R}$ of $R$ is a simple, regular, right and left self-injective ring of Type II.

(c) The natural map center $(D) \rightarrow \operatorname{center}(\bar{R})$ is an isomorphism.

Proof. (a) and (b) are given by Proposition 1.1.

(c) It is clear that the natural map center $(D) \rightarrow \operatorname{center}(R)$ is an isomorphism. Since the maps $\operatorname{center}\left(R_{k}\right) \rightarrow \operatorname{center}\left(R_{k+1}\right)$ are isomorphisms for all $k$, we conclude from Corollary 2.7 that the natural map center $(R) \rightarrow \operatorname{center}(\bar{R})$ is an isomorphism. Thus the natural map center $(D) \rightarrow \operatorname{center}(\bar{R})$ must be an isomorphism.

COROLlary 2.9. Let $F$ be any field. Then there exists a simple, regular, right and left self-injective ring $\bar{R}$ of Type II such that center $(\bar{R}) \cong F$.

Proof. Set $D=F$ and $n(k)=2^{k}$ for all $k$, and construct $\bar{R}$ as in Theorem 2.8 .

We close this section with an example which shows that Theorem 2.6 and Corollary 2.7 may fail if we do not assume that center $\left(R_{i}\right) \subseteq \operatorname{center}\left(R_{j}\right)$ whenever $R_{i} \subseteq R_{j}$. 
EXAMPLE 2.10. There exists a simple regular ring $R$ such that

(a) $R$ is the direct limit of a sequence $R_{1} \rightarrow R_{2} \rightarrow \cdots$ of simple artinian rings and ring maps.

(b) There exists a unique rank function $N$ on $R$.

(c) The $N$-completion $\bar{R}$ of $R$ is a simple, regular, right and left self-injective ring of Type II.

(d) center $(R) \cong \boldsymbol{R}$ but $\operatorname{center}(\bar{R}) \cong \boldsymbol{C}$.

Proof. (a) Set $s(n)=2^{n(n+1) / 2}$ and $R_{n}=M_{s(n)}(C)$ for all $n=$ $1,2, \cdots$. Define $R$-algebra maps $\phi_{n}: R_{n} \rightarrow R_{n+1}$ according to the rule

$$
\phi_{n}(x)=\left(\begin{array}{llllll}
x & & & & \\
& x & & & \\
& & & & \\
& & \cdot & & \\
& & & & \\
& & & x & \\
& & & & \bar{x}
\end{array}\right) \text {, }
$$

where $\bar{x}$ denotes the conjugate (not transposed) of the matrix $x$. Set $R=\lim R_{n}$, and for all $n$ let $\psi_{n}: R_{n} \rightarrow R$ be the natural map.

(b) $\overrightarrow{\text { For }}$ each $n$, there is a unique rank function $P_{n}$ on $R_{n}$, given by the rule $P_{n}(x)=\operatorname{rank}(x) / s(n)$. Observing that $P_{n+1} \phi_{n}=P_{n}$ for all $n$, we see that there is a rank function $N$ on $R$ such that $N \psi_{n}=P_{n}$ for all $n$. Since the $P_{n}$ are unique, $N$ must be unique.

(c) is given by Proposition 1.1.

(d) It is clear that center $(R) \cong R$. Since $N$ is a rank function, we may identify $R$ with its image in $\bar{R}$. Let $\bar{N}$ be the natural extension of $N$ to $\bar{R}$.

For all $n$, let $\theta_{n}: C \rightarrow R_{n}$ be the natural isomorphism of $C$ onto the center of $R_{n}$. Given any $x \in C$, we claim that the sequence $\left\{\psi_{n} \theta_{n}(x)\right\} \subseteq R$ is Cauchy with respect to $N$. For each $n$, we see that $\theta_{n+1}(x)-\phi_{n} \theta_{n}(x)$ is a diagonal matrix with 0 for the first $s(n+1)-s(n)$ diagonal entries and $x-\bar{x}$ for the remaining $s(n)$ diagonal entries, whence $\operatorname{rank}\left(\theta_{n+1}(x)-\phi_{n} \theta_{n}(x)\right) \leqq s(n)$. Consequently,

$$
\begin{aligned}
N\left(\psi_{n+1} \theta_{n+1}(x)-\psi_{n} \theta_{n}(x)\right) & =N \psi_{n+1}\left(\theta_{n+1}(x)-\phi_{n} \theta_{n}(x)\right) \\
& =P_{n+1}\left(\theta_{n+1}(x)-\phi_{n} \theta_{n}(x)\right) \leqq s(n) / s(n+1)=1 / 2^{n+1}
\end{aligned}
$$

As a result, we see for all $k>n$ that

$$
\begin{aligned}
N\left(\psi_{k} \theta_{l}(x)-\psi_{n} \theta_{n}(x)\right) & \leqq \sum_{j=n}^{k-1} N\left(\psi_{j+1} \theta_{j+1}(x)-\psi_{j} \theta_{j}(x)\right) \\
& \leqq \sum_{j=n}^{k-1} 1 / 2^{j+1}<1 / 2^{n}
\end{aligned}
$$


Therefore $\left\{\psi_{n} \theta_{n}(x)\right\}$ is indeed Cauchy with respect to $N$, hence there is a unique element $\gamma(x) \varepsilon \bar{R}$ such that $\psi_{n} \theta_{n}(x) \rightarrow \gamma(x)$ in the $\bar{N}$-metric. Inasmuch as $N\left(\psi_{n} \theta_{n}(x)-\psi_{k} \theta_{k}(x)\right)<1 / 2^{n}$ for all $k>n$, we see that $\bar{N}\left(\psi_{n} \theta_{n}(x)-\gamma(x)\right) \leqq 1 / 2^{n}$ for all $n$.

We now have a map $\gamma: C \rightarrow \bar{R}$, and it is clear that $\gamma$ is an injective ring map. We claim that $\gamma(\boldsymbol{C})=\operatorname{center}(\bar{R})$.

In order to prove that $\gamma(\boldsymbol{C}) \cong \operatorname{center}(\bar{R})$, it suffices to show that for any $x \in C, \gamma(x)$ commutes with any $y \in R$. Choose $k$ such that $y \in \psi_{k}\left(R_{k}\right)$. Given any $n \geqq k$, we have $y=\psi_{n}\left(z_{n}\right)$ for some $z_{n} \in R_{n}$. Since $\theta_{n}(x)$ commutes with $z_{n}$, we see that $\psi_{n} \theta_{n}(x)$ commutes with $y$. Taking limits, we find that $\gamma(x)$ commutes with $y$, as desired.

Finally, consider any $w \in \operatorname{center}(\bar{R})$. There exists $x \in R_{n}$ for some $n$ such that $\bar{N}\left(\psi_{n}(x)-w\right)<1 / 8$. According to Corollary 2.4, there exist elements $y \in R_{n}$ and $z \in \operatorname{center}\left(R_{n}\right)$ such that $(x-z) R_{n} \lesssim$ $(x y-y x) R_{n}$, whence $P_{n}(x-z) \leqq P_{n}(x y-y x)$. Since $w$ commutes with $\psi_{n}(y)$, we obtain

$$
\begin{aligned}
P_{n}(x-z) \leqq P_{n}(x y-y x) & =N\left(\psi_{n}(x) \psi_{n}(y)-\psi_{n}(y) \psi_{n}(x)\right) \\
& =\bar{N}\left(\left(\psi_{n}(x)-w\right) \psi_{n}(y)-\psi_{n}(y)\left(\psi_{n}(x)-w\right)\right) \\
& \leqq 2 \bar{N}\left(\psi_{n}(x)-w\right)<1 / 4 .
\end{aligned}
$$

In addition, we have $z=\theta_{n}(t)$ for some $t \in \boldsymbol{C}$, hence

$$
\bar{N}\left(\psi_{n}(x)-\psi_{n} \theta_{n}(t)\right)=N \psi_{n}(x-z)=P_{n}(x-z)<1 / 4 .
$$

Recalling that $\bar{N}\left(\psi_{n} \theta_{n}(t)-\gamma(t)\right) \leqq 1 / 2^{n}$, we find that

$$
\begin{array}{r}
\bar{N}(w-\gamma(t)) \leqq \bar{N}\left(w-\psi_{n}(x)\right)+\bar{N}\left(\psi_{n}(x)-\psi_{n} \theta_{n}(t)\right)+\bar{N}\left(\psi_{n} \theta_{n}(t)-\gamma(t)\right) \\
<(1 / 8)+(1 / 4)+\left(1 / 2^{n}\right)<1 .
\end{array}
$$

Consequently, $w-\gamma(t)$ is a noninvertible element of the field center $(\bar{R})$, hence $w-\gamma(t)=0$. Thus $w \in \gamma(\boldsymbol{C})$.

Therefore $\gamma(\boldsymbol{C})=$ center $(\bar{R})$ as claimed, so that $\boldsymbol{C} \cong \operatorname{center}(\bar{R})$.

3. Factor rings. This section is concerned with calculating the centers of factor rings of regular, right self-injective rings.

Definition. For any ring $R$, we use $B(R)$ to stand for the Boolean algebra of all central idempotents of $R$. If $R$ is regular and right self-injective, then $B(R)$ is complete by [6, Proposition 4.1].

LEMMA 3.1. Let $R$ be a regular, right self-injective ring, and let $X \subseteq R$. Let $Y \subseteq B(R)$, and set $f=\vee Y$. If $x e=0$ for all $x \in X$ and all $e \in Y$, then $x f=0$ for all $x \in X$. 
Proof. Let $J$ be the right annihilator of $X$, and note that $(R / J)_{R}$ is nonsingular. According to [6, Proposition 4.1], $\sum_{e s Y} e R$ is an essential right $R$-submodule of $f R$. Inasmuch as $\sum_{e \varepsilon Y} e R \leqq J$, we conclude that $f R \leqq J$, so that $x f=0$ for all $x \in X$.

LEMMA 3.2. Let $R$ be a regular, right self-injective ring. Given any $x \in R$, there exist elements $y \in R$ and $f \in B(R)$ such that $f x \in$ center $(R)$ and $(x y-y x) e \neq 0$ for all nonzero $e \leqq 1-f$ in $B(R)$.

Proof. Set $Y=\{e \in B(R) \mid(x t-t x) e=0$ for all $t \in R\}$ and $f=\vee Y$. According to Lemma 3.1, $(f x) t-t(f x)=(x t-t x) f=0$ for all $t \in R$, hence $f x \in \operatorname{center}(R)$.

Let $W$ be the set of those $g \in B(R)$ for which there exists an element $t \in R$ such that $(x t-t x) e \neq 0$ for all nonzero $e \leqq g$ in $B(R)$. We claim that every nonzero $h \leqq 1-f$ in $B(R)$ must lie above some nonzero member of $W$.

Now $h \geqq f$ and so $h \notin Y$, hence $(x t-t x) h \neq 0$ for some $t \in R$. Setting $k=\vee\{e \in B(R) \mid(x t-t x) e=0\}$, we see by Lemma 3.1 that $(x t-t x) k=0$. Then $h \not k$, hence $g=h-h k$ is a nonzero member of $B(R)$. It is clear that $g \leqq h$, and that $(x t-t x) e \neq 0$ for all nonzero $e \leqq g$ in $B(R)$, whence $g \in W$.

Thus every nonzero $h \leqq 1-f$ in $B(R)$ lies above some nonzero member of $W$, as claimed. Consequently, we infer that there exist orthogonal idempotents $g_{i} \in W$ such that $\vee g_{i}=1-f$. For each $i$, there exists an element $y_{i} \in R$ such that $\left(x y_{i}-y_{i} x\right) e \neq 0$ for all nonzero $e \leqq g_{2}$ in $B(R)$. Since $R$ is right self-injective, there exists $y \in R$ such that $y g_{i}=y_{\imath} g_{\imath}$ for all $i$. Given any nonzero $e \leqq 1-f$ in $B(R)$, we have $e g_{i} \neq 0$ for some $i$, whence $(x y-y x) e g_{i}=\left(x y_{i}-y_{i} x\right) e g_{i} \neq$ 0 . Therefore $(x y-y x) e \neq 0$ for all nonzero $e \leqq 1-f$ in $B(R)$.

THEOREM 3.3. Let $R$ be a regular, right self-injective ring, and let $P$ be a minimal prime ideal of $R$. Then the natural map center $(R) \rightarrow \operatorname{center}(R / P)$ is surjective.

Proof. Given any $x \in R$ such that $\bar{x} \in \operatorname{center}(R / P)$, we must show that $\bar{x}=\bar{z}$ in $R / P$ for some $z \in \operatorname{center}(R)$. According to Lemma 3.2, there exist $y \in R$ and $f \in B(R)$ such that $f x \in \operatorname{center}(R)$ and $(x y-y x) e \neq 0$ for all nonzero $e \leqq 1-f$ in $B(R)$.

Since $\bar{x} \in \operatorname{center}(R / P)$, we obtain $x y-y x \in P$. According to [3, Theorem 2.3], $P=[P \cap B(R)] R$, from which it follows that $x y-y x=$ $g(x y-y x)$ for some $g \in P \cap B(R)$. Consequently, $(x y-y x)(1-g)=0$, which implies that $(1-g)(1-f)=0$. Thus $1-f=g(1-f) \in P$, and so $x-f x \in P$.

Therefore we have $f x \in \operatorname{center}(R)$ such that $\bar{x}=\overline{f x}$ in $R / P$. 
Definition. For any ring $R$, we use $B S(R)$ to stand for the set of all maximal ideals of the Boolean algebra $B(R)$.

LEMMA 3.4. Let $R$ be a regular, right self-injective ring which is Type $\mathrm{I}_{n}$ for some $n$. For any $M \in B S(R), R / M R$ is a simple artinian ring, and the natural map center $(R) \rightarrow \operatorname{center}(R / M R)$ is surjective.

Proof. According to [3, Theorem 2.3], $M R$ is a minimal prime ideal of $R$, hence Theorem 3.3 says that the natural map center $(R) \rightarrow$ center $(R / M R)$ is surjective. Now $R \cong M_{n}(S)$ for some abelian, regular, right self-injective ring $S$, hence $R / M R \cong M_{n}(S / Q)$ for some prime ideal $Q$ of $S$. Inasmuch as $S$ is strongly regular, $S / Q$ is a division ring, whence $R / M R$ is simple artinian.

Proposition 3.5. Let $R$ be a regular, right self-injective ring of Type $\mathrm{I}_{f}$. Given any $x \in R$, there exist elements $y \in R$ and $z \in$ center $(R)$ such that $(x-z) R \lesssim(x y-y x) R$.

Proof. According to [6, Corollary 6.5], there exist regular, right self-injective rings $R_{1}, R_{2}, \cdots$ such that $R \cong \Pi R_{n}$ and each $R_{n}$ is Type $\mathrm{I}_{n}$. Consequently, there exist orthogonal central idempotents $e_{1}, e_{2}, \cdots \in B(R)$ such that $\vee e_{n}=1$ and each $e_{n} R$ is Type $\mathrm{I}_{n}$.

Let $X$ be the set of those $f \in B(R)$ for which there exist elements $y, a, b \in R$ and $z \in \operatorname{center}(R)$ such that $f(x-z)=f a(x y-y x) b$. We claim that any nonzero $g \in B(R)$ must lie above some nonzero member of $X$.

Now $g e_{n} \neq 0$ for some $n$, hence there exists $M \in B S(R)$ such that $g e_{n} \notin M$. Since $e_{n} \notin M$, we see that $M \cap e_{n} R \in B S\left(e_{n} R\right)$ and that $e_{n} R /\left(M \cap e_{n} R\right) e_{n} R \cong R / M R$. Consequently, it follows from Lemma 3.4 that $R / M R$ is a simple artinian ring, and that the natural map center $(R) \rightarrow \operatorname{center}(R / M R)$ is surjective. Applying Corollary 2.4 to the element $\bar{x} \in R / M R$, we obtain elements $y^{\prime} \in R / M R$ and $z^{\prime} \in$ center $(R / M R)$ such that $\left(\bar{x}-z^{\prime}\right)(R / M R) \lesssim\left(\bar{x} y^{\prime}-y^{\prime} \bar{x}\right)(R / M R)$. Thus $\bar{x}-z^{\prime}=a^{\prime}\left(\bar{x} y^{\prime}-y^{\prime} \bar{x}\right) b^{\prime}$ for some $a^{\prime}, b^{\prime} \in R / M R$. Now there exist elements $y, a, b \in R$ such that $\bar{y}=y^{\prime}, \bar{a}=a^{\prime}$, and $\bar{b}=b^{\prime}$, and there exists $z \in \operatorname{center}(R)$ such that $\bar{z}=z^{\prime}$.

Thus $x-z-a(x y-y x) b \in M R$, from which it follows that

$$
x-z-a(x y-y x) b=h[x-z-a(x y-y x) b]
$$

for some $h \in M$. Since $g \notin M$ and $h \in M$, we see that $f=g(1-h)$ is not in $M$, whence $f$ is a nonzero member of $B(R)$ such that $f \leqq g$. In addition, $f[x-z-a(x y-y x) b]=0$, so that $f \in X$. Thus the 
claim holds.

Inasmuch as every nonzero member of $B(R)$ lies above a nonzero member of $X$, we infer that there exist orthogonal idempotents $f_{i} \in X$ such that $\vee f_{i}=1$. For each $i$, there exist elements $y_{i}, a_{i}$, $b_{i} \in R$ and $z_{i} \in \operatorname{center}(R)$ such that $f_{i}\left(x-z_{i}\right)=f_{i} a_{i}\left(x y_{i}-y_{i} x\right) b_{i}$. Since $R$ is right self-injective, there exist elements $y, a, b, z \in R$ such that $y f_{i}=y_{i} f_{i}, a f_{i}=a_{i} f_{i}, \quad b f_{i}=b_{i} f_{i}$, and $z f_{i}=z_{i} f_{i}$ for all $i$. Then $f_{i}(x-z)=f_{i} a(x y-y x) b$ for all $i$, hence we see by Lemma 3.1 that $x-z=a(x y-y x) b$. Thus $(x-z) R \lesssim(x y-y x) R$. Since $z f_{i}=z_{i} f_{i} \in$ center $(R)$ for all $i$, we conclude from Lemma 3.1 that $z \in \operatorname{center}(R)$.

THEOREM 3.6. Let $R$ be a regular, right self-injective ring of Type $\mathrm{I}_{f}$. If $K$ is any two-sided ideal of $R$, then the natural map center $(R) \rightarrow \operatorname{center}(R / K)$ is surjective.

Proof. Consider any $x \in R$ such that $\bar{x} \in \operatorname{center}(R / K)$. According to Proposition 3.5, there exist $y \in R$ and $z \in \operatorname{center}(R)$ such that $(x-z) R \lesssim(x y-y x) R$. Since $R / K$ is a flat left $R$-module, it follows that $(\bar{x}-\bar{z})(R / K) \lesssim(\bar{x} \bar{y}-\bar{y} \bar{x})(R / K)$. Since $\bar{x} \in \operatorname{center}(R / K)$, we obtain $\bar{x} \bar{y}=\bar{y} \bar{x}$, and consequently $\bar{x}=\bar{z}$.

The centers of the other standard Type II examples may now be calculated using Theorem 3.6, as follows.

TheORem 3.7. Let $D_{1}, D_{2}, \cdots$ be division rings, let $n(1)<n(2)<$ .. be positive integers, and set $R_{k}=M_{n(k)}\left(D_{k}\right)$ and $F_{k}=\operatorname{center}\left(R_{k}\right)$ for all k. Set $R=\Pi R_{k}$, and let $M$ be any maximal two-sided ideal of $R$ which contains $\oplus R_{k}$. Then $R / M$ is a simple, regular, right and left self-injective ring of Type II, and center $(R / M) \cong$ $\left(\Pi F_{k}\right) /\left[M \cap\left(\Pi F_{k}\right)\right]$.

Proof. Since each $R_{k}$ is a regular, right self-injective ring of Type $\mathrm{I}_{n(k)}$, [6, Corollary 11.10$]$ says that $R / M$ is a simple, regular, right and left self-injective ring of Type II. According to [6, Corollary 6.5], $R$ is Type $\mathrm{I}_{f}$, hence Theorem 3.6 says that the natural map center $(R) \rightarrow \operatorname{center}(R / M)$ is surjective. Observing that center $(R)=$ $\Pi F_{k}$, we conclude that $\operatorname{center}(R / M) \cong\left(\Pi F_{k}\right) /\left[M \cap\left(\Pi F_{k}\right)\right]$.

Unlike the Type II examples obtained from completions, the rings $R / M$ in Theorem 3.7 do not have completely arbitrary centers, as the following corollary (of Theorem 3.6) shows.

Corollary 3.8. Let $R_{1}, R_{2}, \cdots$ be simple artinian rings, set $R=\Pi R_{k}$, and let $M$ be any maximal two-sided ideal of $R$ which 
contains $\oplus R_{k}$. Then center $(R / M)$ is either finite or uncountable.

Proof. Set $F_{k}=\operatorname{center}\left(R_{k}\right)$ for all $k$, and note that center $(R)=$ $\Pi F_{k}$. Note also that $P=M \cap\left(\Pi F_{k}\right)$ is a prime ideal of $\Pi F_{k}$ which contains $\oplus F_{k}$. By [6, Corollary 6.5], $R$ is Type $\mathrm{I}_{f}$, hence Theorem 3.6 says that the natural map center $(R) \rightarrow \operatorname{center}(R / M)$ is surjective. Thus center $(R / M) \cong\left(\Pi F_{k}\right) / P$.

Since $\Pi F_{k}$ is a commutative regular ring, all of its prime ideals are maximal, hence it follows that $P$ is a minimal prime ideal of $\Pi F_{k}$. Consequently, [3, Proposition 3.3] says that there exists an ultrafilter $\mathscr{F}$ on the index set $N=\{1,2, \cdots\}$ such that

$$
P=\left\{x \in \Pi F_{k} \mid\left\{k \in N \mid x_{k}=0\right\} \in \mathscr{F}\right\} .
$$

Thus $\left(\Pi F_{k}\right) / P$ is an ultraproduct of the $F_{k}$. Since $\oplus F_{k} \subseteq P$, we see that $\mathscr{F}$ is a nonprincipal ultrafilter on $N$.

Now if the set $\left\{k \in N \mid F_{k}\right.$ is finite $\}$ belongs to $\mathscr{F}$, then we see by $[2$, Lemmas $3.7,3.11]$ that $\left(\Pi F_{k}\right) / P$ is either finite or uncountable. On the other hand, if the set $\left\{k \in N \mid F_{k}\right.$ is infinite $\}$ belongs to $\mathscr{F}$, then we see by $[2$, Corollaries $1.10,3.14]$ that $\left(\Pi F_{k}\right) / P$ is uncountable. Therefore in all cases $\operatorname{center}(R / M) \cong\left(\Pi F_{k}\right) / P$ is either finite or uncountable.

The following example, and the basic idea for its proof, was suggested by Handelman in correspondence.

EXAMPLE 3.9. There exists a simple regular ring $R$ such that

(a) $R$ is the direct limit of a sequence $R_{1} \rightarrow R_{2} \rightarrow \cdots$ of simple artinian rings and ring maps.

(b) There exists a unique rank function $N$ on $R$.

(c) The $N$-completion $\bar{R}$ of $R$ is a simple, regular, right and left self-injective ring.

(d) There do not exist simple artinian rings $S_{1}, S_{2}, \cdots$ such that $\bar{R}$ is isomorphic to a factor ring of $\Pi S_{n}$.

Proof. Set $D=\boldsymbol{Q}$ and $n(k)=2^{k}$ for all $k=1,2, \cdots$, and construct $R$ as in Theorem 2.8. Then (a), (b), (c) hold, and center $(\bar{R}) \cong \boldsymbol{Q}$.

(d) Suppose that there do exist simple artinian rings $S_{1}, S_{2}, \ldots$ such that $\bar{R} \cong\left(\Pi S_{n}\right) / M$ for some maximal two-sided ideal $M$ of $\Pi S_{n}$. Since $\bar{R}$ is Type II, we see that $\bar{R} \not S_{n}$ for all $n$, whence $\bigoplus S_{n} \subseteq M$. Then Corollary 3.8 says that the center of $\left(\Pi S_{n}\right) / M$ is either finite or uncountable. Since center $(\bar{R})$ is countably infinite, this is impossible.

In view of Example 3.9, we ask whether the simple, regular, 
right and left self-injective rings $R / M$ of Theorem 3.7 can be obtained as in Theorem 2.8. More generally, can every simple, regular, right and left self-injective ring of Type II be obtained as in Theorem 2.8? The following example of von Neumann [12] indicates that the answer to this second question is probably negative.

Set $D=C$, choose positive integers $n(1)<n(2)<\cdots$ such that $n(k) \mid n(k+1)$ for all $k$, and construct $\bar{R}$ as in Theorem 2.8. Let $S$ be the "regular ring" of a complex $W^{*}$-factor of Type $\mathrm{II}_{f}$ (denoted $U(M)$ in [12, $\S 5])$. As indicated in [12, $\S 5], S$ is a continuous regular ring. It is not hard to check that $S$ is simple, and [11, Theorem 7.9] says that $S$ is right and left self-injective. By [11, Theorem 5.1], $S$ is directly finite, from which we infer that $S$ is Type II. However, [12, Theorem E] says that $\bar{R} \not S$.

This example shows that there exists a simple, regular, right and left self-injective ring $S$ of Type II such that $S$ cannot be obtained as in Theorem 2.8 in case $D$ is a field (since then $D \cong C$ ). It still might be possible to obtain $S$ as in Theorem 2.8 with some noncommutative $D$, but this seems unlikely.

\section{REFERENCES}

1. J.H. Alexander, Approximately finite geometries and their coordinate rings, $\mathrm{Ph}$.

D. Dissertation (1957), Univ. of Tennessee.

2. J.L. Bell and A.B. Slomson, Models and Ultraproducts, Amsterdam (1971), NorthHolland.

3. K.R. Goodearl, Prime ideals in regular self-injective rings. II, J. Pure Applied Algebra, 3 (1973), 357-373.

4. - Simple regular rings and rank functions, Math. Annalen, 214 (1975). 267-287.

5. - Completions of regular rings, Math. Annalen, 220 (1976), 229-252.

6. K.R. Goodearl and A.K. Boyle, Dimension theory for nonsingular injective modules, Memoirs American Math. Soc., No. 177 (1976).

7. K.R. Goodearl and D. Handelman, Simple self-injective rings, Communications in Algebra, 3 (1975), 797-834.

8. I. Halperin, Regular rank rings, Canad. J. Math., 17 (1965), 709-719.

9. D. Handelman, Completions of rank rings, Canad. Math. Bull., 20 (1977), 199-205.

10. N. Jacobson, Theory of Rings, New York (1943) American Math. Soc.

11. Y. Utumi, On continuous rings and self-injective rings, Trans. Amer. Math. Soc., 118 (1965), 158-173.

12. J. von Neumann, The nonisomorphism of certain continuous rings, Ann. of Math., 67 (1958), 485-496.

13. Continuous Geometry, Princeton (1960), Princeton Univ. Press.

Received August 15, 1977 and in revised form September 19, 1977. This research was partially supported by a National Science Foundation grant.

UNIVERSITY OF UTAH

Salt Lake City, UT 84112 



\section{PACIFIC JOURNAL OF MATHEMATICS}

\section{EDITORS}

RICHARD ARENS (Managing Editor)

University of California

Los Angeles, CA 90024

Charles W. Curtis

University of Oregon

Eugene, OR 97403

C. C. MOORE

University of California

Berkeley, CA 94720

\section{J. DugundJI}

Department of Mathematics

University of Southern California

Los Angeles, CA 90007

R. FinN and J. Milgram

Stanford University

Stanford, CA 94305

\section{ASSOCIATE EDITORS}
E. F. BECKENBACH
B. H. NeumanN
F. WOLF
K. YosHIDA

\section{SUPPORTING INSTITUTIONS}

\author{
UNIVERSITY OF BRITISH COLUMBIA \\ CALIFORNIA INSTITUTE OF TECHNOLOGY \\ UNIVERSITY OF CALIFORNIA \\ MONTANA STATE UNIVERSITY \\ UNIVERSITY OF NEVADA, RENO \\ NEW MEXICO STATE UNIVERSITY \\ OREGON STATE UNIVERSITY \\ UNIVERSITY OF OREGON
}

\author{
UNIVERSITY OF SOUTHERN CALIFORNIA \\ STANFORD UNIVERSITY \\ UNIVERSITY OF HAWAII \\ UNIVERSITY OF TOKYO \\ UNIVERSITY OF UTAH \\ WASHINGTON STATE UNIVERSITY \\ UNIVERSITY OF WASHINGTON
}

The Supporting Institutions listed above contribute to the cost of publication of this Journal, but they are not owners or publishers and have no responsibility for its content or policies.

Mathematical papers intended for publication in the Pacific Journal of Mathematics should be in typed form or offset-reproduced, (not dittoed), double spaced with large margins. Please do not use built up fractions in the text of the manuscript. However, you may use them in the displayed equations. Underline Greek letters in red, German in green, and script in blue. The first paragraph or two must be capable of being used separately as a synopsis of the entire paper. Items of the bibliography should not be cited there unless absolutely necessary, in which case they must be identified by author and journal, rather than by item number. Manuscripts, in triplicate, may be sent to any one of the editors. Please classify according to the scheme of Math. Reviews, Index to Vol. 39. All other communications should be addressed to the managing editor, or Elaine Barth, University of California, Los Angeles, California, 90024.

50 reprints to each author are provided free for each article, only if page charges have been substantially paid. Additional copies may be obtained at cost in multiples of 50 .

The Pacific Journal of Mathematics is issued monthly as of January 1966. Regular subscription rate: $\$ 72.00$ a year (6 Vols., 12 issues). Special rate: $\$ 36.00$ a year to individual members of supporting institutions.

Subscriptions, orders for numbers issued in the last three calendar years, and changes of address should be sent to Pacific Journal of Mathematics, 103 Highland Boulevard, Berkeley, California, 94708. Older back numbers obtainable from Kraus Periodicals Co., Route 100, Millwood, NY 10546.

PUBLISHED BY PACIFIC JOURNAL OF MATHEMATICS, A NON-PROFIT CORPORATION

Printed at Kokusai Bunken Insatsusha (International Academic Printing Co., Ltd.). 8-8, 3-chome, Takadanobaba, Shinjuku-ku, Tokyo 160, Japan.

Copyright (C) 1978 by Pacific Journal of Mathematics Manufactured and first issued in Japan 


\section{Pacific Journal of Mathematics}

\section{Vol. 76, No. $2 \quad$ December, 1978}

Stephanie Brewster Brewer Taylor Alexander, Local and global convexity in complete Riemannian manifolds ...........................

Claudi Alsina i Català, On countable products and algebraic convexifications of probabilistic metric spaces ...............................

Joel David Berman and George Grätzer, Uniform representations of

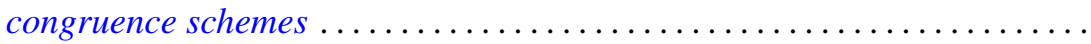

Ajit Kaur Chilana and Kenneth Allen Ross, Spectral synthesis in

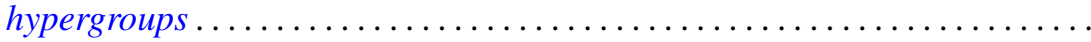

David Mordecai Cohen and Howard Leonard Resnikoff, Hermitian quadratic forms and Hermitian modular forms . .........................

Frank Rimi DeMeyer, Metabelian groups with an irreducible projective

representation of large degree .............................

Robert Ellis, The Furstenberg structure theorem .....................

Heinz W. Engl, Random fixed point theorems for multivalued mappings .......

William Andrew Ettling, On arc length sharpenings ..................

Kent Ralph Fuller and Joel K. Haack, Rings with quivers that are trees........

Kenneth R. Goodearl, Centers of regular self-injective rings ...............

John Gregory, Numerical algorithms for oscillation vectors of second order

differential equations including the Euler-Lagrange equation for

symmetric tridiagonal matrices.

Branko Grünbaum and Geoffrey Shephard, Isotoxal tilings

Myron Stanley Henry and Kenneth Leroy Wiggins, Applications of

approximation theory to differential equations with deviating

arguments

Mark Jungerman, The non-orientable genus of the n-cube .

Robert Richard Kallman, Only trivial Borel measures on $S_{\infty}$ are

quasi-invariant under automorphisms ................

Joyce Longman and Michael Rich, Scalar dependent algebras in the

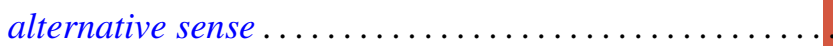

Richard A. Mollin, The Schur group of a field of characteristic zero ........ 471

David Pokrass, Some radical properties of rings with $(a, b, c)=(c, a, b) \ldots 479$

Margaret Shay and Paul Ruel Young, Characterizing the orders changed by

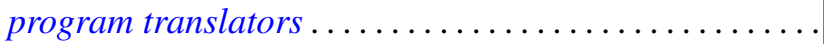

Jerrold Norman Siegel, On the structure of $B_{\infty}(F), F$ a stable space...

Surjeet Singh, (hnp)-rings over which every module admits a basic

submodule...

A. K. Snyder, Universal interpolating sets and the Nevanlinna-Pick property in

Banach spaces of functions...

Jeffrey D. Vaaler, On the metric theory of Diophantine approximation ... 